\title{
THE
}

1993

\section{Chiral exponents of the square-lattice frustrated XY model: A Monte Carlo transfer-matrix calculation}

Enzo Granato

M. P. Nightingale

University of Rhode Island, nightingale@uri.edu

Follow this and additional works at: https://digitalcommons.uri.edu/phys_facpubs

Terms of Use

All rights reserved under copyright.

\section{Citation/Publisher Attribution}

Granato, E., \& Nightingale, M. P. (1993). Chiral exponents of the square-lattice frustrated $X Y$ model: A Monte Carlo transfer-matrix calculation. Physical Review B, 48(10), 7438-7444. doi: 10.1103/ PhysRevB.48.7438

Available at: http://dx.doi.org/10.1103/PhysRevB.48.7438

This Article is brought to you for free and open access by the Physics at DigitalCommons@URI. It has been accepted for inclusion in Physics Faculty Publications by an authorized administrator of DigitalCommons@URI. For more information, please contact digitalcommons-group@uri.edu. 


\title{
Chiral exponents of the square-lattice frustrated $X Y$ model: A Monte Carlo transfer-matrix calculation
}

\author{
Enzo Granato \\ Laboratório Associado de Sensores e Materiais, Instituto Nacional de Pesquisas Espaciais, \\ 12.225 São José dos Campos, São Paulo, Brazil \\ M. P. Nightingale \\ Department of Physics, University of Rhode Island, Kingston, Rhode Island 02881
}

(Received 4 May 1993)

\begin{abstract}
Thermal and chiral critical exponents of the fully frustrated $X Y$ model on a square lattice are obtained from a finite-size scaling analysis of the free energy of chiral domain walls. Data were obtained by extensive Monte Carlo transfer-matrix computations for infinite strips of widths up to 14 lattice spacings. Two transfer matrices were implemented, one for each of the two principal lattice directions. The results of both are consistent, but the critical exponents differ significantly from the pure Ising values. This is in agreement with other recent Monte Carlo simulations. Our results also support the identification of the critical behavior of this model with that along the line of transitions of simultaneous ordering or becoming critical of Ising and planar rotor degrees of freedom in the $X Y$-Ising model studied recently.
\end{abstract}

\section{INTRODUCTION}

There has been considerable interest, both experimentally and theoretically, in phase transitions of twodimensional, fully frustrated $X Y$ models. Most studies have been motivated by their relevance to Josephson junction arrays in a magnetic field, where these models describe the superconducting-to-normal transition at half a flux quantum per plaquette ${ }^{1}$ but there are also interesting theoretical questions regarding the identification of the universality class. ${ }^{2-14}$

In the standard $X Y$ model without frustration the relevant symmetry is the continuous $U(1)$ symmetry which, as is well-known, is responsible for a Kosterlitz-Thouless phase transition. The fully frustrated $X Y$ model has a richer behavior with a low-temperature phase with critical fluctuations associated with the U(1) symmetry, accompanied by a broken, discrete $Z_{2}$ symmetry. In principle, there are two different ordering scenarios: ordering can take place in two stages via separate $X Y$ and Ising transitions, or both symmetries can be broken or become critical simultaneously, which yields a single transition, presumably in a new universality class.

On a square lattice the model can be defined by the Hamiltonian

$$
H=-\sum_{\langle i j\rangle} J_{i j} \cos \left(\theta_{i}-\theta_{j}\right),
$$

where $J_{i j}=J(J>0)$ for horizontal rows and $J_{i j}= \pm J$ for alternating vertical columns. Owing to the presence of an odd number of antiferromagnetic bonds in each plaquette, the model is fully frustrated. This leads to a double degeneracy of the ground state, which is of course accompanied by an additional continuous degen- eracy, a manifestation of the $\mathrm{U}(1)$ symmetry. One can introduce an Ising-like order parameter $\chi_{p}$, the local chirality, which measures the sense of rotation of a spin of unit length $\mathbf{s}=(\cos \theta, \sin \theta)$ along the sides of a given plaquette $p$. In the ground state there is antiferromagnetic order of the local chiralities $\left(\chi_{p}= \pm 1\right)$.

Early Monte Carlo simulations led to the conclusion that the chiral order parameter of the fully frustrated $X Y$ model has pure Ising model critical exponents, ${ }^{3-9}$ but more recent ${ }^{10-13}$ estimates of the exponents have differed significantly from the Ising values. In particular, predictions were made for the values of chiral critical exponents of the fully frustrated $X Y$ model. These predictions are based on results for the $X Y$-Ising model, ${ }^{11,14}$ which is expected to describe the critical behavior in these systems. These ideas are supported by recent Monte Carlo simulations. ${ }^{12,13}$ However, in view of results that suggest pure Ising critical exponents, and in the absence of precise agreement among the more recent estimates, the current state of affairs is unsatisfactory from a computational point of view. The additional numerical results presented in this paper may serve to help settle the issue.

We report results of extensive numerical calculations of the chiral domain wall free energy of the fully frustrated $X Y$ model in an infinite strip geometry. Because of the continuous nature of the phase variables of this model, the transfer-matrix is intractable for numerically exact computation of its eigenvalues. We therefore use the Monte Carlo transfer-matrix method ${ }^{15}$ to obtain the free energy from the largest eigenvalue of the transfer matrix. Exploiting the anisotropy of the Hamiltonian (1) we use two different implementations, a "horizontal" (i.e., column-to-column) and "vertical" (i.e., row-to-row) transfer matrix. These approaches yield results in reasonable mutual agreement. From a finite-size scaling analy- 
sis of data for strips of widths up to 14 lattice spacings, we have estimated the thermal exponent $\nu$, the exponent $\eta$ associated with the Ising-like order-order correlation function, and the critical temperature $T_{c}$. In particular, the result obtained for $\nu$ is quite insensitive of the estimate of $T_{c}$, as will be discussed in more detail below. We summarize our estimates (with error estimates in parentheses): $\nu=0.80(4), \eta=0.38(3)$, and $T_{c}=0.454(4)$. Within the errors, these numbers are in agreement, with a Monte Carlo simulation using similar finite-size scaling analysis $^{12}$ and they are also consistent with other Monte Carlo simulations. ${ }^{13}$ However, our value of $\nu$ disagrees with the estimate of $\nu=1$ by Thijssen and Knops, ${ }^{10}$ who also used the Monte Carlo transfer-matrix method, but a different method to estimate $\nu$; their deviating estimate is likely to be an artifact of their fitting procedure ${ }^{16} \mathrm{On}$ the other hand, our value of $\eta$ is in very good agreement with their estimate. Moreover, our results appear to support the identification of the critical behavior of the fully frustrated $X Y$ model with the line of single transitions in the $X Y$-Ising model studied recently. ${ }^{11,14}$

\section{MONTE CARLO TRANSFER MATRIX}

The Monte Carlo transfer-matrix method introduced by Nightingale and Blöte, ${ }^{15}$ is particularly useful when, as is the case in the model under consideration, the continuous nature of the spin variables does not allow a numerically exact diagonalization of the transfer matrix. The method is a stochastic version of the well-known power method of calculating the dominant eigenvalue of a matrix or integral kernel. More specifically, the dominant eigenvector $\psi^{0}$ is approximated by a Monte Carlo time average over weighted walkers representing row (or column) configurations. The basic idea is that the (weighted) frequency of occurrence of a particular row (or column) configuration, say $S=\left(\mathbf{s}_{1}, \mathbf{s}_{2}, \ldots, \mathbf{s}_{L}\right)$, is proportional to the magnitude of the corresponding component $\psi_{S}^{0}$ of the dominant eigenvector.

The key elements of the algorithm are the following. Walkers are generated in subsequent generations labeled by an index $t$. The generation at time $\boldsymbol{t}$ consists of a sequence of a number of $r_{t}$ walkers $\left[\left(S_{1, t}, w_{1, t}\right),\left(S_{2, t}, w_{2, t}\right), \ldots,\left(S_{r_{t}, t}, w_{r_{t}, t}\right)\right]$, where the $S_{i, t}$ are row (or column) configurations of the form $S$ introduced above, and the $w_{i, t}>0$ are statistical weights. This sequence represents a (generally extremely) sparse vector $\psi$ with components

$$
\psi_{S}=\sum_{i=1}^{r_{t}} \delta_{S, S_{i}} w_{i, t}
$$

where $\delta$ is the Kronecker $\delta$ function. One can write

$$
\psi_{S^{\prime}}^{\prime}=\sum_{S} T\left(S^{\prime} \mid S\right) \psi_{S}=\sum_{S} P\left(S^{\prime} \mid S\right) D_{S} \psi_{S}
$$

where $D_{S}=\sum_{S^{\prime}} T\left(S^{\prime} \mid S\right)$ and $P\left(S^{\prime} \mid S\right)=T\left(S^{\prime} \mid S\right) / D_{S}$. Since by construction $P$ is a stochastic matrix, multiplication by the transfer matrix of a vector of the form $\psi$ can be implemented as a stochastic process with transitions from $S$ to $S^{\prime}$ with probability $P\left(S^{\prime} \mid S\right)$. That is, to update generation $t$ to $t+1$, new walker states are sampled with probability $P\left(S_{t+1} \mid S_{t}\right)$, while in each transition the weight of walker $i$ is updated to $w_{i, t+1}=$ $D_{S} w_{i, t} / c_{t+1}$. For reasons of efficiency the weights are kept close to unity by duplicating walkers with great statistical weights and by eliminating walkers with low weights. To ensure that $r_{t+1}$ remains close to its initial value $r_{0}$, one can choose $c_{t+1}=\lambda_{t} r_{t} / r_{0}$, where $\lambda_{t}$ is a moving or cumulative average estimate of the dominant transfer-matrix eigenvalue. Assuming that the generation counter $t$ is reset to unity upon equilibration, the largest eigenvalue can be estimated from a sequence of $T$ generations as

$$
\lambda=\sum_{t=1}^{T} c_{t+1} W_{t+1} / \sum_{t=1}^{T} W_{t},
$$

where $W_{t^{\prime}}=\sum_{i} w_{i, t^{\prime}}$ denotes the total statistical weight of all walkers of generation $t^{\prime}$. It should be noted that this estimator of the dominant eigenvalue suffers from a bias due to the correlations of the population control constants $c_{t}$ with the weights $W_{t}$. These correlations have the effect of suppressing large contributions to the estimator (4) and enhancing small ones. ${ }^{17}$ As a result, the estimator tends to underestimate the exact transfer-matrix eigenvalue, but this effect can be reduced by choosing a large target number of walkers, $r_{0}$. Further algorithmic details, such as how to correct for this bias without increasing the average population size, and an alternative, sometimes better choice of the population control constant $c_{t}$ can be found in Refs. 18-20. In applying this method to the fully frustrated $X Y$ model, we have performed extensive calculations using, typically, $r_{0}=30000$ walkers and 150000 Monte Carlo steps which corresponds to $4.5 \times 10^{9}$ attempts per site.

To apply this Monte Carlo method it is necessary to sample configurations $S^{\prime}$ from the distribution $P\left(S^{\prime} \mid S\right)$ for arbitrary $S$ and to evaluate the quantity $D_{S}$ at each elementary Monte Carlo step. One way to do this is to factor the transfer matrix in a way that amounts to building up the square lattice by, e.g., first adding bonds perpendicular to the transfer direction and then adding new sites and bonds along the transfer direction. The algorithm given above is then applied to both factors in succession. The first of these operations corresponds to multiplication by a diagonal matrix. This leaves the row (or column) configurations unchanged while the weight factors are calculated trivially: they consist of one term each. The second matrix factor, which adds $L$ new sites, has a direct product form. Sampling and reweighting problems in this case reduce to simple one-site problems. In principle, this method works, but it yields an inefficient Monte Carlo process: the simple factorization generates row (or column) configurations with variables that are too weakly correlated in the sense that, as it is added to the lattice, each variable is sampled independently from a distribution with direct correlations only to one other nearest-neighbor variable. True many-site correlations are subsequently recovered via multiplication by 
strongly fluctuating weight factors. This, however, causes strong fluctuations in the number of walkers, which results in frequent elimination and duplication of walkers and enhances correlations between walkers within each generation, which reduces the overall Monte Carlo sampling efficiency.

The alternative we have used in this paper requires helical boundary conditions. ${ }^{15}$ In that case the lattice can be constructed by repetition of identical elementary steps, each of which adds one site at a time. This implies that the states $S^{\prime}$ and $S$ in the transition matrix $P\left(S^{\prime} \mid S\right)$ consist of lattice sites all of which coincide with the exception of one site. Consequently only a small number of states $S^{\prime}$ can be reached from any given state $S$, i.e., $P$ is sparse in this case. The degrees of freedom that are added will have direct correlations with those at two neighboring lattice sites, one in the horizontal and one in the vertical direction for the square lattice. In principle, given an approximate dominant eigenvector, one can transform the transfer matrix and incorporate even more correlations from the onset, and thus reduce the fluctuations of the weights and eigenvalue estimates. ${ }^{20-22} \mathrm{We}$ will return to this later on.

Use of helical boundary yields a more efficient Monte Carlo process, but the method has the disadvantage of producing a lattice with a surface that has a step defect, as is unavoidable when one cuts across a screw. One would expect the presence of this defect to lead to unnecessary corrections to scaling, which may adversely affect finite-size convergence, but in practice this appears not to be a serious problem.

In fact, the method used in this paper requires use of a transfer matrix of somewhat more complicated form than the one of Eq. (3), in that this equation has to be replaced by

$$
\psi_{S^{\prime \prime}}^{\prime \prime}=\sum_{S^{\prime}, S} T^{(1)}\left(S^{\prime \prime} \mid S^{\prime}\right) T^{(2)}\left(S^{\prime} \mid S\right) \psi_{S}
$$

Although this is no real complication-one can simply devise a stochastic process with steps alternating in correspondence to the two matrices $T^{(1)}$ and $T^{(2)}$-it has prevented our use of approximate trial eigenvectors to reduce the noise of the stochastic process. In principle, this variance reduction scheme works as follows. Equation (3) can be replaced by the equivalent equation

$$
\hat{\psi}_{S^{\prime}}^{\prime}=\sum_{S} \hat{T}\left(S^{\prime} \mid S\right) \hat{\psi}_{S}
$$

where $\hat{T}\left(S^{\prime} \mid S\right)=\gamma_{S^{\prime}} T\left(S^{\prime} \mid S\right) / \gamma_{S}$ and $\psi$ and $\psi^{\prime}$ are similarly similarity transformed. As long as the components of $\gamma$ are all of one sign and do not vanish, the same Monte Carlo method can be applied to $\hat{T}$ instead of $T$. This approach satisfies the following zero-variance principle: in the ideal limit where $\gamma$ is the dominant left eigenvector of $T$, the largest eigenvalue of $T$ can be estimated with vanishing statistical error. More realistically, as this limit is approached the variance of the Monte Carlo process decreases. In practical applications, one chooses a trial vector $\gamma\left(p_{1}, p_{2}, \ldots\right)$ which depends on variational param- eters $p_{i}$. These are optimized by minimization of the variance over $S$ of $\sum_{S^{\prime}} \gamma_{S^{\prime}} T\left(S^{\prime} \mid S\right) / \gamma_{S}$ with states selected with probability proportional to $\gamma_{S}^{2}$. This minimization is accomplished approximately by minimizing the variance over a relatively small set of states $S$ generated by Monte Carlo. ${ }^{23}$ For a transfer matrix of the form of Eq. 5 the same process is still possible in principle, but it is more complicated. In fact there are two alternatives. One can define a Kronecker-product-like, two-site transfer matrix $T^{(12)}\left(S^{\prime \prime}, S^{\prime} \mid S^{\prime}, S\right)=T^{(1)}\left(S^{\prime \prime} \mid S^{\prime}\right) T^{(2)}\left(S^{\prime} \mid S\right)$ and base the Monte Carlo process on the derived stochastic matrix $P\left(S^{\prime \prime}, S^{\prime} \mid S^{\prime}, S\right) \propto \gamma_{S^{\prime \prime}, S^{\prime}}^{(12)} T^{(12)}\left(S^{\prime \prime}, S^{\prime} \mid S^{\prime}, S\right) / \gamma_{S^{\prime}, S}^{(12)}$, where ideally $\gamma^{(12)}$ is the dominant left eigenvector of $T^{(12)}$. We note that the pairs $\left(S^{\prime \prime}, S^{\prime}\right)$ and $\left(S^{\prime}, S\right)$ differ at two lattice sites, and therefore this approach requires simultaneous sampling of two site variables, which renders the algorithm unnecessarily slow. The alternative is to employas we have done in this paper-a matrix product of two single-site transfer matrices rather than a single twosite transfer matrix, but this approach requires two trial vectors. In terms of these, one defines $\hat{T}^{(1)}\left(S^{\prime} \mid S\right)=$ $\gamma_{S^{\prime}}^{(1)} T^{(1)}\left(S^{\prime} \mid S\right) / \gamma_{S}^{(2)}$, and $\hat{T}_{S^{\prime}, S}^{(2)}=\gamma_{S^{\prime}}^{(2)} T^{(2)}\left(S^{\prime} \mid S\right) / \gamma_{S}^{(1)}$. It is straightforward to construct a transformed process which again has a zero variance principle. This time it requires that $\gamma^{(1)}$ be the dominant left eigenvector of the product matrix $T^{(1)} T^{(2)}$ and that $\gamma^{(2)}$ be the dominant left eigenvector of $T^{(2)} T^{(1)}$. Again adjustable parameters of $\gamma^{(1)}$ and $\gamma^{(2)}$ can be chosen by minimization of the (appropriately weighted) sum of the variances of $\sum_{S^{\prime}} \gamma_{S^{\prime}} T\left(S^{\prime} \mid S\right) / \gamma_{S}$ and $\sum_{S^{\prime}} \gamma_{S^{\prime}} T\left(S^{\prime} \mid S\right) / \gamma_{S}$. In contrast with the alternative of a two-site transfer matrix, the presence of two matrices slows down the algorithm only in the initial stage of parameter optimization with this approach.

\section{CHIRAL DOMAIN WALL FREE ENERGY}

For an infinite strip of width $L$, the reduced free energy $f$ per lattice site can be obtained from the largest transfer matrix eigenvalue, $\lambda(L, K)$, via the relation $f=-\ln \lambda$, where $K=J / k_{\mathrm{B}} T$ is the reduced coupling constant. For any given $L$ this quantity, $f$, depends on the choice of boundary conditions. By suitable choice of the latter, as specified in detail below, the chiral domain wall free energy can be obtained from the free energy difference, denoted by $\Delta f$. For finite-size analysis, a convenient quantity is the domain wall energy per $L$ lattice units of length:

$$
\Delta F(K, L)=L^{2} \Delta f(K, L)
$$

Since the fully frustrated $X Y$ model is spatially anisotropic, one can devise two different types of boundary conditions to compute the domain wall free energy. These two types are associated with two different transfer matrices obtained by choosing the transfer direction to be either horizontal or vertical, as shown in Fig. 1. If the transfer direction is horizontal [Fig. 1(a)], one is forced to use helical boundary conditions with a pitch of two measured in lattice units, so as to match up the vertical 


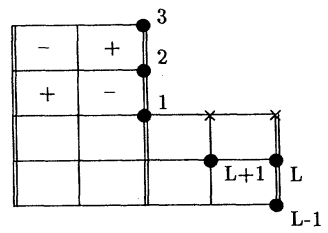

a)

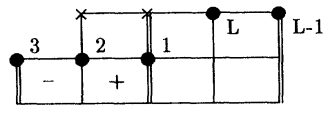

b)

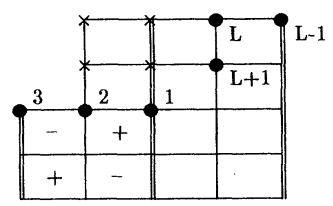

c)

FIG. 1. Schematic of the process of building up the infinite strip using a transfer matrix. This can be implemented using a Monte Carlo transfer matrix along the horizontal direction with double helical boundary conditions (a) or along the vertical direction with single (b) or double (c) helical boundary conditions. At each step a new configuration is obtained from the previous one, indicated by $\bullet$, by adding a new spin $\times$ through a Monte Carlo process and relabeling the sites. The \pm indicates the antiferromagnetic pattern of the chirality in the ground state.

antiferromagnetic bonds, which have a periodicity of two in the transfer direction. This is the construction used in Ref. 10. As indicated in Fig. 1(a), only strips with $L$ even will match the antiferromagnetic pattern of the local chiralities $\chi_{p}$ in the ground state; for strips with odd $L$ the boundary conditions will introduce a chiral domain wall along the infinite horizontal direction. Calculation of the domain wall energy requires that the boundary conditions be varied at constant $L$. With the horizontal transfer matrix this can be done approximately only, and we chose to use the difference of the free energy computed directly for $L$, and the free energy obtained by linear interpolation between sizes $L-1$ and $L+1$. This still

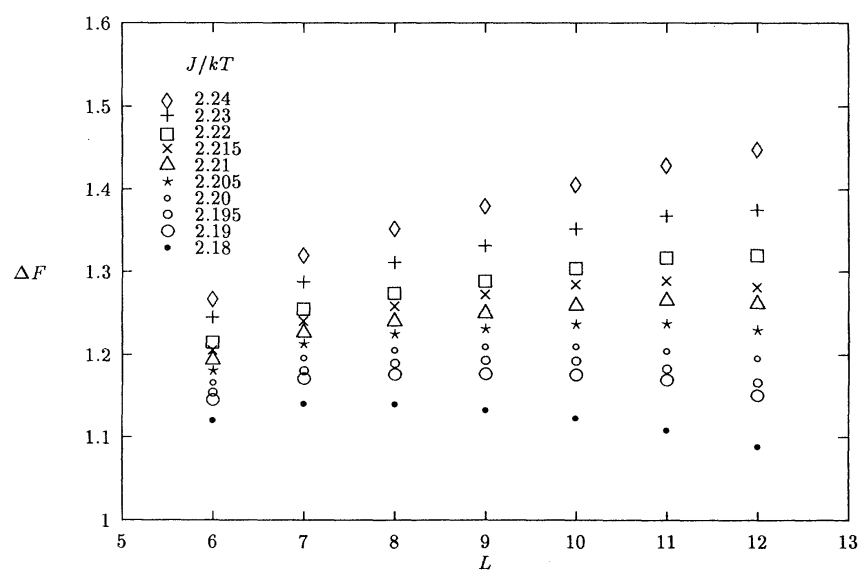

FIG. 2. Data for the chiral domain wall free energy $\Delta F(K, L)$, obtained from horizontal Monte Carlo transfer-matrix calculations, in a small range around $T_{c}$.

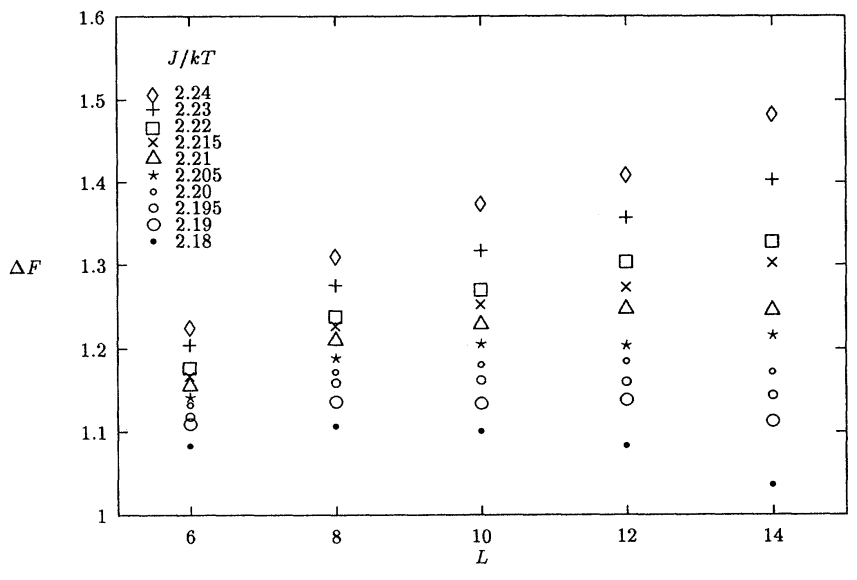

FIG. 3. Chiral domain wall free energy $\Delta F(K, L)$, obtained from vertical Monte Carlo transfer matrix calculations, for the same couplings $K$ of Fig. 2.

leaves two possibilities, depending on whether $L$ is odd or even. As an alternative we also performed calculations using a vertical transfer matrix illustrated in Fig. 1. In this case one has a choice between helical boundary conditions with a pitch of one or two lattice units. For each value of $L$ precisely one of these two boundary conditions will force the presence of a domain wall and this offers a convenient way to determine the chiral domain free energy without interpolation.

Calculations of the free energy using the horizontal and vertical transfer matrices were performed as a function of strip width in a very narrow range of couplings $\Delta K=0.04$ around the estimate of the critical coupling obtained by Monte Carlo simulations of Lee, Kosterlitz, and Granato. ${ }^{12}$ Note that the range we use is about ten times smaller than the range used in Ref. 10. The data for the chiral domain wall free energy using both implementations of the transfer matrix are shown in Figs. 2 and 3.

\section{FINITE-SIZE SCALING ANALYSIS}

To determine the critical temperature and critical exponents, we make use of the following finite-size scaling relation for the domain wall free energy

$$
\Delta F(K, L)=A\left(L^{1 / \nu} \Delta K\right)
$$

where $A$ is a scaling function and $\Delta K=K-K_{c}$ is the deviation of the coupling constant from its critical value $K_{c}$. For sufficiently small values of its argument, the scaling function can be expanded as

$$
\Delta F(K, L)=a_{0}+a_{1} L^{1 / \nu} \Delta K+a_{2}\left(L^{1 / \nu} \Delta K\right)^{2}+\cdots,
$$

which shows that $\Delta F(K, L)$ is constant as as function of $L$ for $K=K_{c}$, but for that value of the coupling constant only. This behavior is apparent in Figs. 2 and 3 for 
at least $L \geq 8$ if we attribute the deviations for smaller system sizes to corrections to scaling. To the extent that the quadratic and higher-order terms in Eq. (9) can be neglected, the critical exponent $\nu$ can be obtained from the condition that $\Delta F(K, L)$ be linear in $L^{1 / \nu}$ for fixed $\Delta K$. In fact, in this approximation, ${ }^{24} \nu$ can be obtained from the slope of a log-log plot of $S=\partial \Delta F(K, L) / \partial K$ vs $L$, which gives $1 / \nu$. From the data of Figs. 2 and 3 , we have obtained $S$ as a function of $L$ for the horizontal and vertical transfer matrix using this procedure. The results are indicated in Fig. 4. The slopes of the straight lines in the log-log plot, corresponding to the results for vertical and horizontal transfer matrices, agree within the errors, as one would expect, providing a check to the consistency of our data. From this plot we can estimate $\nu=0.80(4)$. It is interesting to note that within the linear approximation-as is the case for the free energy barrier in the histogram of chirality in the Monte Carlo simulations, ${ }^{12}$ where a similar finite-size scaling is possible $e^{25}$ - the estimate of the critical temperature $T_{c}=1 / K_{c}$ and the estimate of the thermal exponent $\nu$ are in fact independent, ${ }^{24}$ but this property is preserved only approximately in our generalized finite-size analysis, where we fitted the domain wall energies to the form of Eq. (9) with as fitting parameters $K_{c}, \nu$, and $a_{i}$ with $i=0,1$, and 2 , as obtained by truncation of the scaling function beyond second order. Note that the correlation function exponent $\eta$ can be obtained from the universal amplitude $a_{0}$ in Eq. (9) using the results of conformal invariance in two dimensions, ${ }^{26} a_{0}=\pi \eta$.

The results of the finite-size scaling based on Eq. (9) fits are summarized in Table $I$ and the corresponding scaling plots are shown in Figs. 5 and 6. The table contains estimates of the statistical errors associated with the least-squares procedure. In some cases the $\chi^{2}$ were too large to be attributable to chance and as consequence these statistical error estimates are to be treated with suspicion. This is also evident from the discrepancies

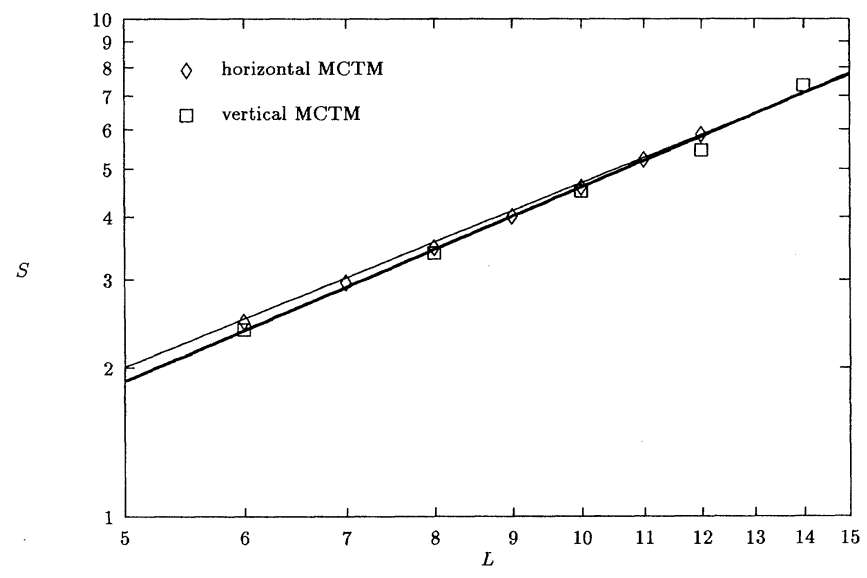

FIG. 4. $S=\partial \Delta F(K, L) / \partial K$ obtained from Monte Carlo transfer-matrix data of Figs. 2 and 3 . The exponent $1 / \nu$ is obtained from the slopes of the linear fit of horizontal (thin line) and vertical (thick line) Monte Carlo transfer-matrix calculations.
TABLE I. Results for critical temperature and critical exponents, obtained from finite-size scaling analysis of domain wall energies. Standard errors are indicated parenthetically. As explained in the text, these standard errors reflect only statistical uncertainties, which are presumably considerably smaller than the errors due to corrections to scaling. The column labeled $L$ indicates which system sizes were used in the fits: $L_{1}, L_{2}(\Delta L)$ stands for sizes from $L_{1}$ to $L_{2}$ in steps of $\Delta L$. Horizontal and vertical transfer matrices are indicated by $v$ and $h$ under the heading transfer. The last column is the $\chi^{2}$ per degree of freedom.

\begin{tabular}{cccccc}
\hline \hline & & & & & \\
$T_{c}$ & $\nu$ & $\eta$ & $L$ & Transfer & $\chi^{2}$ \\
\hline $0.4544(3)$ & $0.77(5)$ & $0.378(3)$ & $10,14(2)$ & $v$ & 11 \\
$0.4555(2)$ & $0.83(4)$ & $0.369(2)$ & $8,14(2)$ & $v$ & 14 \\
$0.4538(2)$ & $0.83(5)$ & $0.394(1)$ & $8,12(2)$ & $h$ & 1 \\
\hline \hline
\end{tabular}

between the various independent estimates. Under the circumstances all we can do is to take the mutual differences of various estimates as (admittedly unsatisfactory) error estimates. Thus we obtain our estimates: $T_{\mathrm{c}}=0.454(3), \nu=0.80(5)$, and $\eta=0.38(2)$. This result for $\nu$ is inconsistent with the pure Ising value of $\nu=1$, but it is in agreement with the result $\nu=0.85(3)$ from a similar finite-size scaling analysis of the free energy barrier in the histogram of chirality from other Monte Carlo simulations. ${ }^{12}$ Our estimate also agrees with a recent estimate $\nu=0.875(35)$ based on a finite-size scaling analysis of correlation functions. ${ }^{13} \mathrm{We}$ therefore conclude that the estimate of $\nu=1$ obtained by Thijssen and Knops ${ }^{10}$ is likely to be an artifact of their fitting procedure.

Recently, the phase diagram of the two-dimensional $X Y$-Ising model defined by the Hamiltonian

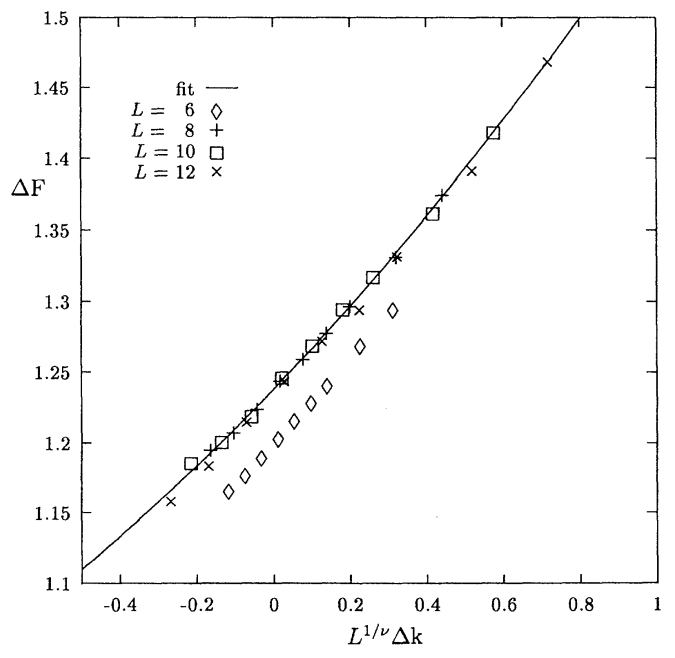

FIG. 5. Scaling plot of domain wall free energy data obtained for the horizontal transfer matrix: $\Delta F$ vs $L^{1 / \nu} \Delta K$. The solid curve is the fitted scaling function $A$, as given by the expansion in Eq. 9. The data for $L=6$ were not used for the fit. 


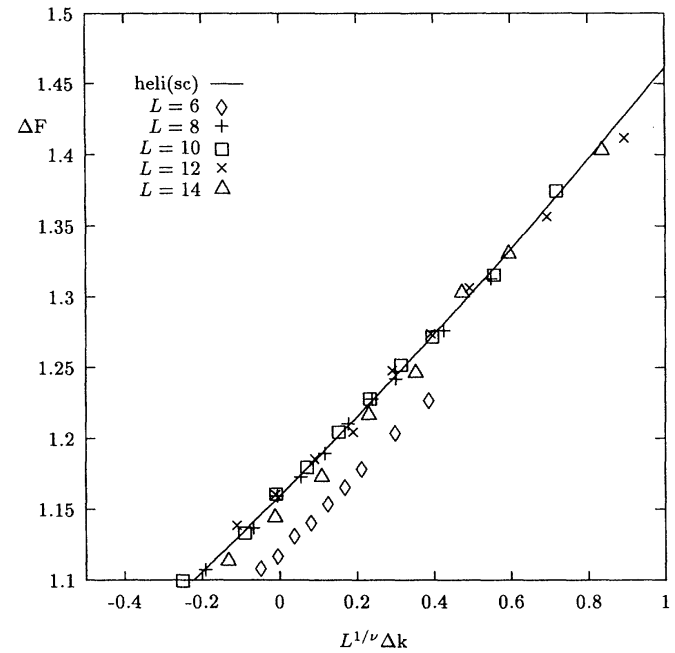

FIG. 6. Same as Fig. 5 for the vertical transfer matrix.

$$
\beta H=-\sum_{\left\langle r r^{\prime}\right\rangle}\left[A\left(1+\sigma_{r} \sigma_{r^{\prime}}\right) \cos \left(\theta_{r}-\theta_{r^{\prime}}\right)+C \sigma_{r} \sigma_{r^{\prime}}\right]
$$

where $\sigma= \pm 1$, has been studied in some detail. ${ }^{11,14}$ This model is expected to describe the critical behavior of a class of systems in which $\mathrm{U}(1)$ and $Z_{2}$ symmetries play a simultaneous role, a class of which the fully frustrated $X Y$ model is a special case. The critical behavior of the square lattice fully frustrated $X Y$ model is represented by the behavior of a particular (unknown) point in the parameter space $(A, C)$ of this model. The phase diagram of the model defined in Eq. (10) consists of three branches, in the ferromagnetic region, joining at $C \approx 0$. Along one of these branches $(C<0)$ the transition takes place at a critical point with simultaneous criticality of $\mathrm{U}(1)$ and $Z_{2}$ order parameters. Along this line, the critical behavior appears to be nonuniversal with $\nu$ varying from 0.76 to 0.84 and $\eta$ varying from 0.25 to 0.5 along a segment of the line. Further away from the branch point, this line of single transition appears to become first order. Our results for the chiral critical exponents of the square lattice fully frustrated $X Y$ obtained by the Monte Carlo transfer-matrix method do in fact appear to be consistent with the critical exponents of a particular point along the line of continuous, single transitions of the $X Y$-Ising model. Once this notion is accepted, it implies that the fully frustrated $X Y$ has a single nonuniversal transition. The nonuniversality of this transition in turn suggests that the critical exponents of the square and triangular lattice fully frustrated $X Y$ models may differ from each other, although they may be quite close. ${ }^{12}$

Besides critical exponents, another important quantity that can be inferred from Monte Carlo transfer matrix calculations, is the central charge $c$, which classifies the possible conformally invariant critical theories. ${ }^{27}$ The central charge is related to the amplitude of the singular part of the free energy per site, at criticality, in the infinite strip by

$$
f\left(K_{c}, L\right)=f_{\infty}+\frac{\pi c}{6 L^{2}}
$$

which is valid asymptotically for large $L$. Fitting the data for $f(K, L)$ closest to the estimated critical temperature $T_{c}$, we obtain $c=1.61(3)$ from the strips of $8 \leq L \leq 14$ using both horizontal and vertical Monte Carlo data. This result agrees with the estimate of the central charge first obtained by Thijssen and Knops, ${ }^{10}$ also using Monte Carlo transfer-matrix calculations, and appears to be significantly larger than $c=3 / 2$, which would be expected if the transition was single, but decoupled. ${ }^{28}$ However, one cannot be certain of this value unless calculations are done at sufficiently large $L$ such that small- $L$ corrections to the above asymptotic expression are negligible. Using only the Monte Carlo transfer-matrix data for $6 \leq L \leq 14$ may not allow us to extrapolate to the $L$ large limit and it is quite likely that this estimate of $c$ is subject to systematic errors. In fact, recent large $L$ calculations for the related $X Y$-Ising model ${ }^{29}$ show a significant decrease of $c$ with increasing $L$. Unfortunately, our data for the fully frustrated $X Y$ model for $L>14$ turn out to be rather noisy. This problem can be remedied in principle by using variance reductions techniques, ${ }^{20-22}$ but as discussed this is complicated in the case of the square lattice fully frustrated $X Y$ model because of the presence of both ferromagnetic and antiferromagnetic bonds. The case of the triangular lattice is more straightforward in this respect.

\section{CONCLUSION}

We have studied the finite-size behavior of the chiral domain wall free energy of the square lattice fully frustrated $X Y$ model on an infinite strip, using the Monte Carlo transfer matrix. From a finite-size scaling analysis of data for strip widths up to 14 lattice spacing, we have estimated the critical temperature and chiral critical exponents. The latter appear to be significantly different from the pure Ising values and in agreement with other recent Monte Carlo simulations. The results are also consistent with the identification of the critical behavior of the fully frustrated $X Y$ model with that of a point of the line of single transitions in the $X Y$-Ising model studied recently. ${ }^{30}$ The value of the central charge $c=1.61(3)$ is found to be consistent with the estimate first obtained by Thijssen and Knops, but we cannot rule out the possibility that all results obtained for this and similar models are skewed by large, slowly decaying corrections to scaling.

\section{ACKNOWLEDGMENTS}

We thank J. M. Kosterlitz, T. Ala-Nissila, and K. Kankaala for many helpful discussions. Monte Carlo sampling of the $X Y$ degrees of freedom was performed with a routine developed in a previous collaboration with Dr. H. W. J. Blöte. Part of the computations were carried out in a Cray XMP with computer time provided 
by the Scientific Computational Center of Finland and their generous support is gratefully acknowledged. This work was supported by Fundação de Amparo à Pesquisa do Estado de São Paulo (FAPESP, Proc. No. 92/0963-
5) and Conselho Nacional de Desenvolvimento Científico e Tecnológico (CNPq) (E.G.) and by the National Science Foundation under Grant Nos. DMR-9214669 and CHE-9203498 (M.P.N.).
${ }^{1}$ See, for example, H.S.J. van der Zant, H.A. Rijken, and J.E. Mooij, J. Low Temp. Phys. 82, 67 (1991), and several articles in Proceedings of the Nato Advanced Research Workshop on Coherence in Superconducting Networks, Delft, 1987, edited by J.E. Mooij and G.B.J. Shön [Physica B 152, 1 (1988)].

2 J. Villain, Phys. C 10, 4793 (1977).

${ }^{3}$ S. Teitel and C. Jayaprakash, Phys. Rev. B 27, 598 (1983).

${ }^{4}$ S. Miyashita and H. Shiba, J. Phys. Soc. Jpn. 53, 1145 (1984).

${ }^{5}$ D.H. Lee, J.D. Joannopoulos, J.W. Negele, and D.P. Landau, Phys. Rev. B 33, 450 (1986).

${ }^{6}$ E. van Himbergen, Phys. Rev. B 33, 7857 (1986).

${ }^{7}$ B. Berge, H.T. Diep, A. Ghazali, and P. Lallemmand, Phys. Rev. B 34, 3177 (1986).

${ }^{8}$ J.M. Thijssen and H.J.F. Knops, Phys. Rev. B 37, 7738 (1988).

${ }^{9}$ G. Grest, Phys. Rev. B 39, 9267 (1989).

${ }^{10}$ J.M. Thijssen and H.J.F. Knops, Phys. Rev. B 42, 2438 (1990).

${ }^{11}$ E. Granato, J.M. Kosterlitz, J. Lee, and M.P. Nightingale, Phys. Rev. Lett. 66, 1090 (1991).

12 J. Lee, J.M. Kosterlitz, and E. Granato, Phys. Rev. B 43 , 11531 (1991).

${ }^{13}$ G. Ramirez-Santiago and J. José, Phys. Rev. Lett. 68, 1224 (1992).

${ }^{14}$ J. Lee, E. Granato, and J.M. Kosterlitz, Phys. Rev. B 44, 4819 (1991); E. Granato, J. Phys. C 20, L215 (1987).

${ }^{15}$ M.P. Nightingale and H.W.J. Blöte, Phys. Rev. Lett. 60, 1562 (1988)

${ }^{16}$ The analysis used by Thijssen and Knops (Ref. 10) consists in extrapolating the finite-size data to the thermodynamic limit and subsequently extracting $\nu$ from the singularity of the extrapolated function. The problem of this approach is that there is no reliable procedure to obtain a function which is singular in the thermodynamic limit as the extrapolation of regular functions computed for finite systems. Procedures using finite-size scaling relations such as the ones we employ avoid this problem.

${ }^{17}$ C.J. Umrigar, M.P. Nightingale, and K.J. Runge, J. Chem.
Phys. 99, 2865 (1993).

${ }^{18}$ J.H. Hetherington, Phys. Rev. A 30, 2713 (1984).

${ }^{19}$ M.P. Nightingale and H.W.J. Blöte, Phys. Rev. B 33, 659 (1986).

${ }^{20}$ M.P. Nightingale, in Finite Size Scaling and Numerical Simulations of Statistical Systems, edited by V. Privman (World Scientific, Singapore, 1990).

${ }^{21}$ M.P. Nightingale and R.G. Caflisch, in Computer Simulation Studies in Condensed Matter Physics, edited by D.P. Landau and H.B. Schüttler (Springer, Berlin, 1988), p. 208.

${ }^{22}$ M.P. Nightingale, in Proceedings of the Third International Conference on Supercomputing, edited by L.P. Kartashev and S.I. Kartashev (International Supercomputing Institute, Inc., Boston, 1988), Vol. I, p. 427.

${ }^{23}$ C.J. Umrigar, K.G. Wilson, and J.W. Wilkins, Phys. Rev. Lett. 60, 1719 (1988); and in Computer Simulation Studies in Condensed Matter Physics: Recent Developments, edited by D.P. Landau, K.K. Mon, and H.B. Schüttler, Springer Proc. Phys. Vol. 33 (Springer, Berlin, 1988).

${ }^{24}$ E. Granato, Phys. Rev. B 45, 2557 (1992), and (unpublished).

${ }^{25}$ J. Lee and J.M. Kosterlitz, Phys. Rev. Lett. 65, 137 (1990); Phys. Rev. B 43, 3265 (1991); J.M. Kosterlitz, J. Lee, and E. Granato, in Computer Simulations Studies in Condensed Matter Physics IV, edited by D.P. Landau, K.K. Mon, and H.-B. Schüttler, Springer Proc. Phys. Vol. 72 (Springer, Berlin, 1993), p. 28.

${ }^{26}$ J.L. Cardy, J. Phys. A 17, L961 (1984).

${ }^{27}$ H.W. Blöte, J.L. Cardy, and M.P. Nightingale, Phys. Rev. Lett. 56, 742 (1986).

${ }^{28}$ O. Foda, Nucl. Phys. B300, 611 (1988).

${ }^{29}$ M.P. Nightingale, E. Granato, and J.M. Kosterlitz (unpublished).

${ }^{30}$ Essentially the same results have also been obtained for a one-dimensional quantum version of the fully frustrated $X Y$ model which can describe the $T=0$ superconductorinsulator transition due to charging effects in a ladder of Josephson junctions at a half a flux quantum per plaquette (Ref. 24). 